\title{
Optimal coronary interventional strategies for in-stent restenosis
}

$\mathrm{E}$ verolimus-eluting stents are the most effective treatment for in-stent restenosis, according to a new network meta-analysis published in The Lancet. The incidence of in-stent restenosis after percutaneous coronary intervention (PCI) for coronary artery disease has declined since the introduction of drug-eluting stents in 2002. Nevertheless, $5-10 \%$ of patients require repeat revascularization for in-stent resentosis after PCI with a drug-eluting stent, as do $20-30 \%$ of patients after PCI with a baremetal stent. The optimal interventional strategy for in-stent restenosis is uncertain, although the latest-generation drug-eluting stents and drug-coated balloon angioplasty have a class Ia recommendation in the ESC clinical guidelines. Accordingly, George Siontis and colleagues performed a network meta-analysis of the available data to compare and rank the current interventional modalities used for treatment of in-stent restenosis.

The researchers identified 27 eligible trials including a total of 5,923 patients. Duration of follow-up ranged from 6 months to 60 months. In $84 \%$ of the patients, angiographic follow-up was available at 6-12 months after the index intervention, and the primary outcome was percent diameter stenosis at this angiographic follow-up. According to the meta-analysis, PCI with everolimuseluting stents was the most effective strategy. Compared with this intervention, the difference in terms of percent diameter stenosis of using a drug-coated balloon was $-9.0 \%$ (95\% CI -15.8 to -2.2$)$, a sirolimus-eluting stent was $-9.4 \%$ (95\% CI -17.4 to -1.4 ), a paclitaxel-eluting stent was $-10.2 \%$ (95\% CI -18.4 to -2.0$)$, vascular brachytherapy was -19.2 (95\% CI -28.2 to -10.4 ), a bare-metal stent was $-23.4 \%$ (95\% CI -36.2 to -10.8$)$, balloon angioplasty was $-24.2 \%$ (95\% CI -32.2 to -16.4 ), and rotablation was $-31.8 \%$ (95\% CI -44.8 to -18.6$)$.
Everolimus-eluting stents were the most effective strategy regardless of whether the restenosis occurred in a bare-metal stent or a drug-eluting stent. The investigators suggest that "the advantage of everolimuseluting stents in this setting might be attributable to the thin-strut profile of its cobalt-chromium platform, the antiproliferative potency of the released limus analogue ... and the biocompatibility and thromboresistant properties of its polymer coating".

Intervention with a drug-coated balloon was ranked as the second-best strategy, although the results did not differ significantly from PCI with either a sirolimus-eluting or a paclitaxel-eluting stent. The researchers note that "although drug-coated balloons had inferior angiographic and clinical effectiveness [to everolimus-eluting stents], they could remain a valuable therapeutic alternative because of their ability to provide favourable results in the absence of an additional metallic stent layer. This layer could be particularly important for patients with recurrent coronary in-stent restenosis." Of note, balloon angioplasty, vascular brachytherapy, rotablation, and PCI with a bare-metal stent "cannot be deemed therapeutic alternatives for treatment of in-stent restenosis" according to the investigators.

In an editorial that accompanied the study publication in The Lancet, Bernard Chevalier from the Institut Cardiovasculaire Paris Sud, France, comments that "Siontis and colleagues have established that drug elution from the stent, with extended spot contact with the restenotic tissue, is better than elution from a balloon, where there is a short but diffuse contact". Chevalier also notes that "the absence of long term follow-up in most drug-eluting stent or drugcoated balloon trials included in these meta-analyses partly limits their clinical usefulness because the drug-eluting stent

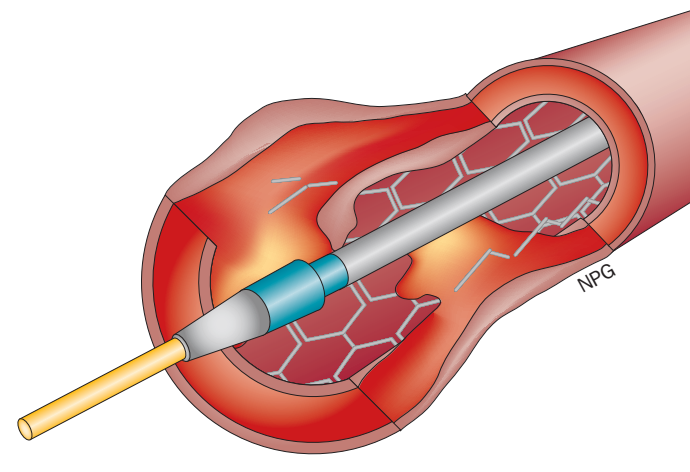

strategy creates a double layer of metal, with possible harmful effects in terms of late failure due to neoatherogenesis".

J. Wouter Jukema, from the Leiden University Medical Center, the Netherlands, and who was not involved in the meta-analysis, agrees that "everolimuseluting stents, followed closely by drugcoated balloons, are the most effective strategies for treatment of restenosis, with the lowest risk of restenosis and repeat revascularizations compared with other treatments. [The meta-analysis] is thus a very welcome confirmation of guidelines and most practices."

A potential limitation of the metaanalysis is that the researchers did not have access to individual patient data and, therefore, the findings have to be considered as average effects. No potential differential effects of available strategies on subgroups of patients or lesions could be identified. Commenting on the findings, George Siontis acknowledges that "several novel technologies have been developed and have not been tested in patients with in-stent restenosis. Drugcoated balloons with limus analogues and fully bioresorbable vascular scaffolds may potentially provide favourable outcomes and need to be evaluated in appropriately designed randomized trials."

Gregory B. Lim

\footnotetext{
Original article Siontis, G. C. M. et al. Percutaneous coronary interventional strategies for treatment of in-stent restenosis: a network meta-analysis. Lancet 386, 655-664 (2015) Further reading Jukema, J. W. et al. Restenosis after PCl. Part 1: pathophysiology and risk factors. Nat. Rev. Cardiol. 9, 53-62 (2012) | Jukema, J. W. et al. Restenosis after PCl. Part 2: prevention and therapy. Nat. Rev. Cardiol. 9, 79-90 (2012)
} 